Classification

Physics Abstracts

$68.55-78.70 \mathrm{C}$

\title{
High resolution X-ray scattering studies of substrates and multilayers
}

\author{
F. E. Christensen \\ Danish Space Research Institute, Lundtoftevej 7, 2800 Lyngby, Denmark \\ (Reçu le 2 octobre 1987, révisé le $1^{\text {er }}$ décembre 1987, accepté le 5 février 1988)
}

\begin{abstract}
Résumé. - On rappelle d'abord le principe des études de rugosités des surfaces à l'aide de la diffusion des rayons X par réflectivité sous incidence rasante; on examine aussi celui des miroirs interférentiels multicouches. A l'aide de cette technique d'étude on montre que le float glass et les tranches de silicium possèdent des défauts importants de planéité, alors que des supports de $\mathrm{SiC}$ superpolis ont une planéité et des rugosités comparables à celles des substrats plans préparés pour les télescopes $\mathrm{X}$. On montre que les défauts de planéité des supports ont des effets notables sur l'efficacité des miroirs X multicouches. Enfin ces études de diffusion $\mathrm{X}$ sous incidence rasante sont employées pour caractériser une multicouche $\mathrm{W} / \mathrm{Si}$ de très bonne qualité préparée sur une tranche de silicium.
\end{abstract}

\begin{abstract}
High resolution X-ray scattering measurements on multilayer substrates and surfaces are reviewed. It is shown that the usual substrates of float glass and Si-wafers are dominated by large scale figure error, whereas samples of super polished $\mathrm{SiC}$ substrates are comparable in flatness and roughness to state-ofthe-art test flats from current $\mathrm{X}$-ray telescope programs. Likewise high resolution X-ray scattering from periodic multilayer structures is reviewed. It is demonstrated that the large scale figure error of the substrate has a profound effect on low resolution measurements. High resolution studies of specific multilayers reveal in a single case of a W/Si multilayer on a Si-wafer an essentially perfect layering.
\end{abstract}

\section{High resolution measurements from substrates and surfaces of multilayers.}

The requirement for high resolution scattering measurements from surfaces is a finely collimated beam of X-rays incident on the surface under a grazing angle of incidence lower than the critical angle of total reflection. Likewise the reflected/scattered beam must be detected with a narrowly collimated detector. The two collimations, before and after the surface, are of the same order of magnitude. There are basically two ways of obtaining this. Either by using narrow slits far apart or to utilize the narrow angular bandpass of perfect crystals of Si. The Si-crystal method is advantageous in several respects. First of all the experiments may be carried out in a compact arrangement. Secondly, letting the X-rays be reflected several times in the channelcut Si-crystals provide a narrow and extremely sharp probe of scattered X-rays. A multipurpose diffractometer based on perfect crystals of $\mathrm{Si}$ is described in detail in reference [1].
The use of this arrangement for high resolution surface scattering was first reported by Matsushita et al. [2] at the photon factory in Japan. They used an additional premonochromator, in a dispersive setting, which simultaneously provided them with a very monochromatic beam. This allows one to use the same arrangement for high resolution X-ray diffraction studies of periodic structures. This is only possible at a synchrotron $\mathrm{X}$-ray source. With a low intensity X-ray source one must use a different crystal arrangement for high resolution studies of periodic structures. This is further discussed later.

The high resolution scattering arrangement is shown in figure 1 . The multiple bounces in the crystals have the effect of exponentiating the single reflection curve of the perfect crystal and, therefore, tails of the resulting reflection curve are greatly diminished providing an extremely sharp resolution probe of the scattered X-rays. Scattering measurements reviewed in the following sections were all done at Fek $\alpha=6.4 \mathrm{keV}$. The actual measurement 


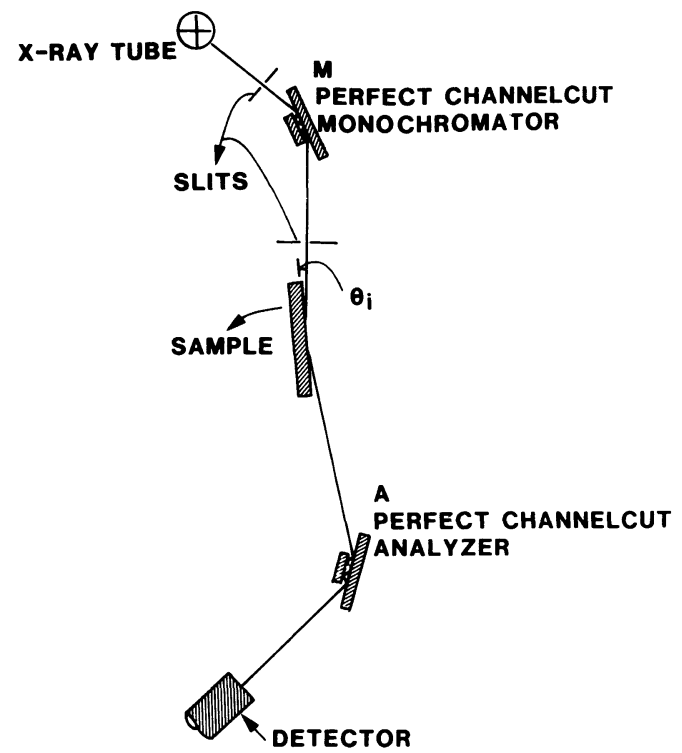

Fig. 1. - Experimental arrangement for the surface scattering experiments.

is performed by rotating the analyser crystal thus detecting the radiation scattered/reflected in different directions from the sample. Scattering data from three different substrates, float glass, Si-wafer and a super-polished $\mathrm{SiC}$ are discussed in the following sections.

An alternative method of measuring the scattering of X-rays from mirrored surfaces have been devised by Mancini and Bilderback [3]. They use the distortion of the diffraction pattern of a $20 \mu$ wide slit to give a fast and integrated picture of the quality of the surface. The method has not been applied to multilayers or substrates.

\section{Relation between high resolution scattering data and surface roughness parameter.}

Two models have been employed to relate high resolution scattering data to physical surface parameters. The first is a facet model [2] which simply assumes that the scattered intensity originates from facets offset slightly from each other. For a continuous surface this gives a relation between scattered intensity and slope errors. The second approach is to use either the scalar theory of roughness induced scattering or the first order vector perturbation theory [7-14]. Both of these predict essentially the same in the socalled smooth surface limit [7-14]. For the scalar theory one obtains [5] :

$$
\begin{gathered}
I(\theta)=\int S\left(\theta_{\mathrm{s}}\right) R_{\mathrm{i}}\left(\theta-\theta_{\mathrm{s}}\right) \mathrm{d} \theta_{\mathrm{s}} \\
S\left(\theta_{\mathrm{s}}\right) \propto G^{2} \mathrm{e}^{-\gamma} \times \\
\times\left[\sin c^{2}(p L)+\frac{\gamma}{L} \int \cos (p \tau) C(\tau) \mathrm{d} \tau\right]
\end{gathered}
$$

where $\theta$ and $\theta_{\mathrm{s}}$ are scattering angles measured from the surface of the sample. $R_{\mathrm{i}}\left(\theta-\theta_{\mathrm{s}}\right)$ is the resolution-curve of the instrument and equation (1) is simply a convolution of the scattering function $S\left(\theta_{\mathrm{s}}\right)$ and the resolution-curve.

The parameters in equation (2) are given by :

$$
\begin{gathered}
\sigma^{2} C(\tau)=\frac{1}{2 L} \int_{-L}^{+L} Z(x) Z(x+\tau) \mathrm{d} x ; \\
L \rightarrow \infty
\end{gathered}
$$

$\sigma$ is the surface height variance. $2 L$ is the illuminated distance along the surface. $Z(x)$ is the surface height profile, and $p$ is given by :

$$
p=k \cdot\left(\cos \theta_{\mathrm{s}}-\cos \theta_{\mathrm{i}}\right) ; k=2 \pi / \lambda
$$

$\theta_{i}$ is the incident angle on the surface and $\lambda$ is the wavelength of the radiation. $\gamma$ and $G$ are given by :

$$
\begin{aligned}
\Delta^{2} & \equiv \gamma=\left(2 k \sigma \sin \theta_{\mathrm{i}}\right)^{2} \\
G & =F \sqrt{R_{\mathrm{f}}\left(\theta_{\mathrm{i}}\right)} \\
F & =\frac{1}{\sin \theta_{\mathrm{i}}} \frac{1-\cos \left(\theta_{\mathrm{i}}+\theta_{\mathrm{s}}\right)}{\sin \theta_{\mathrm{i}}+\sin \theta_{\mathrm{s}}}
\end{aligned}
$$

$R_{\mathrm{f}}\left(\theta_{\mathrm{i}}\right)$ is the Fresnel reflectivity at $\theta_{\mathrm{i}}$.

Equation (2) contains two terms in the square brackets. The first term is the specular term. $\left(\sin c^{2}(p L)\right)$ is essentially a delta function in the specular direction). The second term is the scattered intensity and it is the Fourier transform of the surface height correlation function. The condition for fulfilling the smooth surface limit differs in the two theories. First order vector theory gives the condition $(k \sigma)^{2}<1$. The scalar theory gives the condition $\Delta^{2}<1$.

Differences between the scalar theory and the first order vector perturbation theory show up in the prefactor of the scattering term of equation (3). This is discussed in reference [14] and some experimental evidence for the superiority of the vector perturbation theory is presented in reference [5].

\section{Scattering from float glass.}

The scattering data [4] from float glass at two incident angles $\left(\theta_{i}=0.1^{\circ}, \theta_{i}=0.2^{\circ}\right)-($ Fig. 2) show that the scattered radiation is spread over a wide angular range of 50 arcsec. This is interpreted according to a facet model. The two peaks in the data are fingerprints of two facets in the surface with an angular misorientation of 20 arcsec leading to 40 arcsec in the analyser crystal offset but there clearly is a continuous transition from one to the other. Approximately $50 \mathrm{~mm}$ of the surface was illuminated. Varying the incident angle leads to varying shadow effects. This shows up as a change of the peak structure when $\theta_{i}$ is changed. 


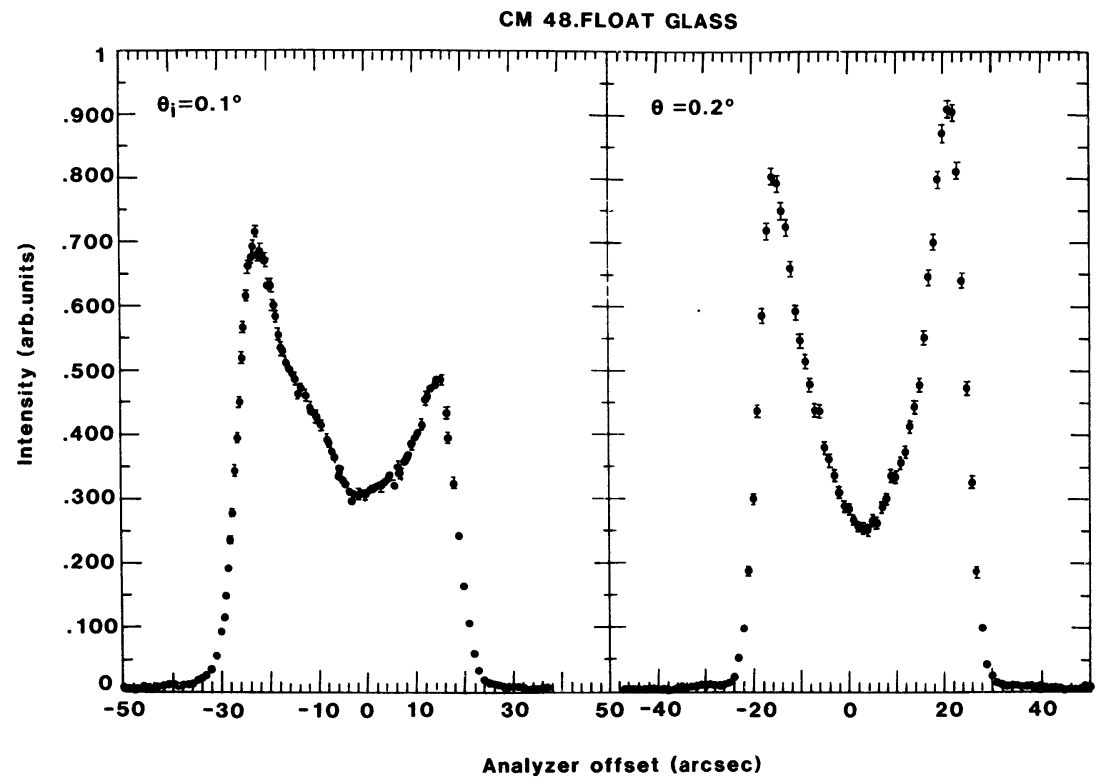

Fig. 2. - Scattering data from float glass at two incident angles : $\theta_{\mathrm{i}}=0.1^{\circ}$ and $\theta_{\mathrm{i}}=0.2^{\circ}$.

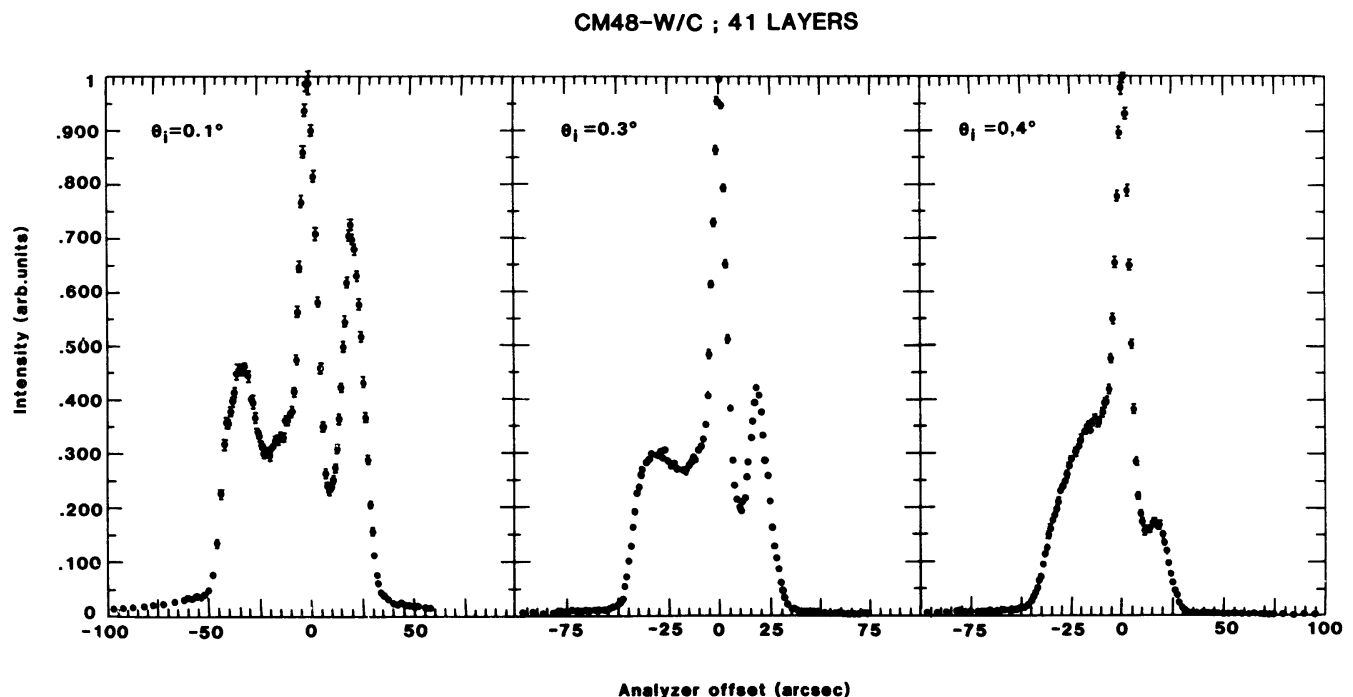

Fig. 3. - Scattering data at $\theta_{\mathrm{i}}=0.1^{\circ}, 0.3^{\circ}$ and $0.4^{\circ}$ from the surface of a W/C multilayer deposited on float glass.

Figure 3 shows scattering data [4] from the surface of a W/C multilayer, deposited on float glass. Data are shown for $\theta_{\mathrm{i}}=0.1^{\circ}, 0.3^{\circ}$ and $0.4^{\circ}$. The illuminated distance at $0.1^{\circ}$ was $50 \mathrm{~mm}$. Again these data are consistent with the continuous variation of the slope of the float glass surface, with three dominating facets illuminated. Two of the peaks almost disappear at $\theta_{\mathrm{i}}=0.4^{\circ}$. This is related to the smaller illuminated spot at $\theta_{\mathrm{i}}=0.4^{\circ}$ as compared to $\theta_{i}=0.1^{\circ}$, so that only one of the major facets contributes.

In accordance with these data Ishikawa et al. [8] have published similar data on angle-resolved X-ray scattering from an optical flat and a W/C multilayer deposited on top of it. They also obtain multifacetted scattering curves but with a sharp central peak as narrow as the instrumental resolution. This is probably due to the small irradiated area. They use a $20 \mu$ wide entrance slit which limits the irradiated length to $\sim 6 \mathrm{~mm}$ at an incident angle of $0.2^{\circ}$. This is considerable smaller than for the data in figures 2 and 3 . The data in figure 3 at an incident angle of $0.4^{\circ}$ resemble the Ishikawa data most consistent with the fact that for these data the irradiated length is the smallest. 


\section{Scattering from Si-wafers.}

Measurements of surface scattering from a Si-wafer and a W/C multilayer deposited on it have been reported by Ishikawa et al. [8]. Recently similar data have been obtained [4] from a W/Si multilayer deposited on a Si-wafer. These data are shown in figure 4 . The data resemble the data from the float glass surface in the sense that there is a multipeaked structure over an angular range of $50 \mathrm{arcsec}$. The data in reference [8] and the data in figure 4 are quite similar and reveal more discontinuous scat-

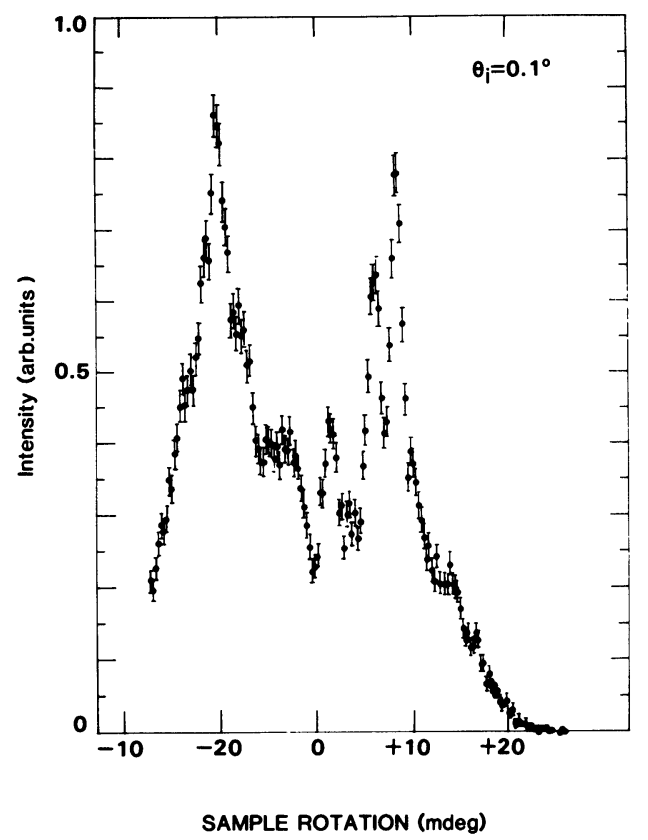

tering curves than the float glass. This may be interpreted physically as being related to the crystal structure of the Si-wafer, whereas the float glass being amorphous, evidently has a smoother variation of the slope of the surface.

It is interesting to note that the smaller irradiated length of the Ishikawa data does not lead to a sharp central peak as is the case for the float glass data. This means that the Si-wafer has many facets offset from one another even over a length scale of $\sim 5 \mathrm{~mm}$.

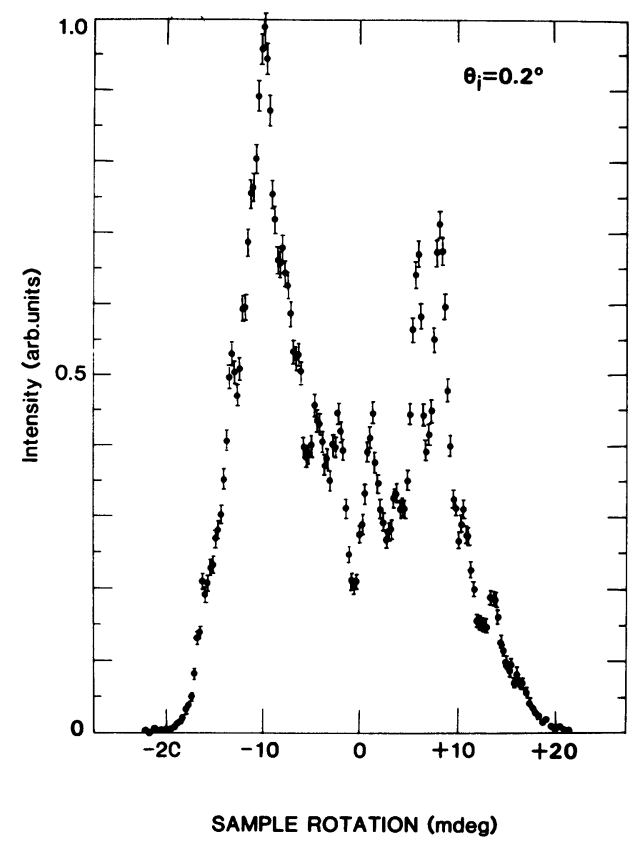

Fig. 4. - Scattering data at $\theta_{\mathrm{i}}=0.1^{\circ}$ and $0.2^{\circ}$ from the surface of a W/Si multilayer deposited on a Si-wafer.

\section{Scattering from superpolished SiC.}

The last example of substrate to be discussed in this review is super-polished SiC. After the super-polishing it may be expected that large scale figure error/slope errors are not present and any scattering is due to roughness on shorter lengthscales the micron-mm range [5]. Measurements of scattering from this surface are shown in figure 5 [4]. In the same figure scattering data from a state-of-the-art standard test flat from the Italian X-ray telescope program SAX are shown for comparison. This test flat is produced by evaporating $500 \AA$ thick Au layer on to a superpolished zerodur glass substrate. Clearly the amount of scattering is extremely low in both cases. The data have been plotted on a log-log scale to show how closely the data follow the resolution function of the instrument. Evidently the $\mathrm{SiC}$ substrate is comparable in smoothness to stateof-the-art polished glass masters for X-ray telescopes.
It must be noted, however, that the SiC-substrate was a circular testflat only $2.5 \mathrm{~cm}$ in diameter. This limits the width of the incident beam which is usefull under grazing incidence. The consequence of this is a loss of intensity and the ratio of peak intensity to background is only $10^{3}$ whereas it is $10^{4}-10^{5}$ for the SAX-standard test flat. It will, therefore, be extremely usefull to perform scattering measurements on larger samples of $\mathrm{SiC}$-substrates. The $\mathrm{SiC}$-substrate which was used for the measurement in figure 5 was kindly provided by Ferranti-Astron, England.

Measuring the specular reflectivity $R$ of smooth surfaces provides a further means of assessing the surface roughness. From first order vector perturbation theory it is known that the deviation between the classical Fresnel curve [12] and the specular reflectivity may be related to the surface roughness via a Debye-Waller factor.

$$
R^{\text {spec }}=R^{\text {Fresnel }} \mathrm{e}^{-\left(\frac{4 \pi \sigma \cos \theta}{\lambda}\right)^{2}}
$$




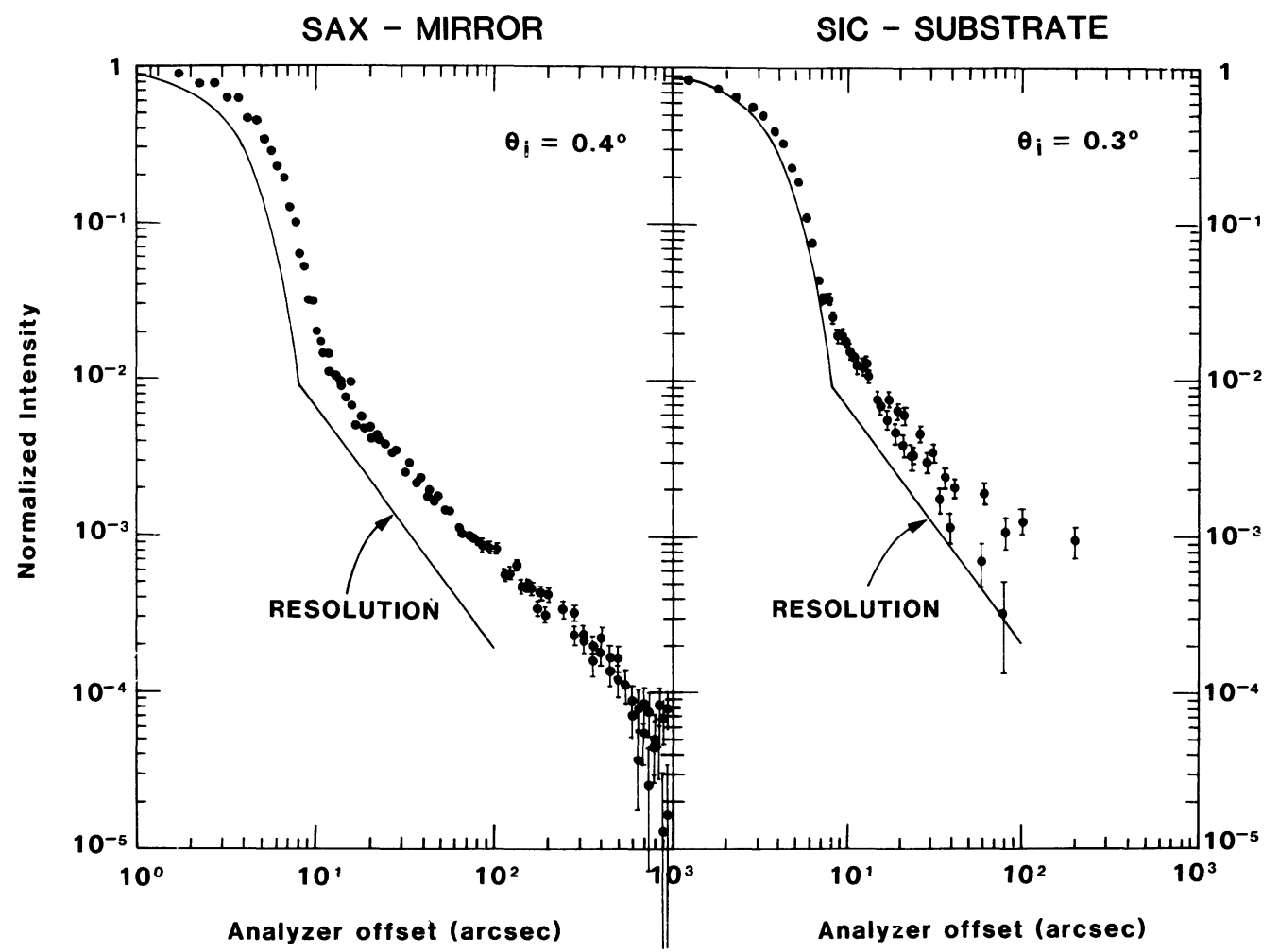

Fig. 5. - Scattering data from polished SiC-substrate $\theta_{i}=0.3^{\circ}$ and from a state-of-the-art standard test flat from the Italian X-ray telescope program SAX. Data are plotted on a log-log scale together with the resolution function of the diffractometer.

Total reflectivity, Si C 02

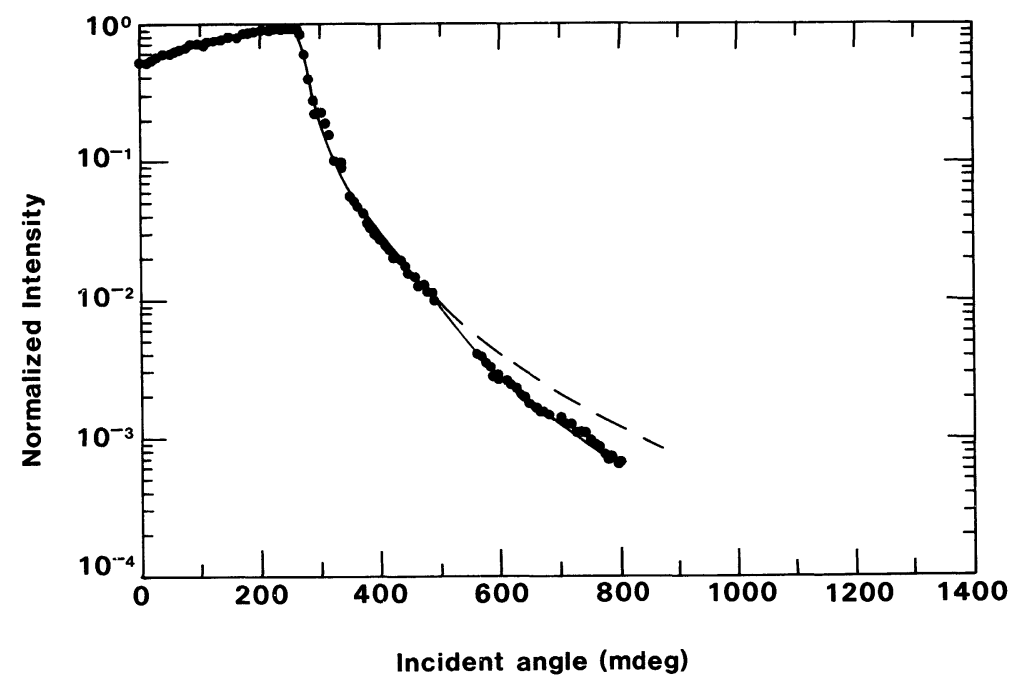

Fig. 6. - Total integrated reflectivity from the polished SiC-substrate and the SAX-standard test flat.

where $\sigma$ is the r.m.s. surface roughness, $\lambda$ is the wavelength of radiation and $\theta$ is the incident angle. Specular reflectivity data have been obtained in a few cases from a polished SiC-substrate [4, 13]. In figure 6 specular reflectivity data are shown for the same $\mathrm{SiC}$-substrate as studied in figure 5. The data in figure 6 were obtained at $\mathrm{FeK} \alpha$ using a Graphite
(002) crystal as monochromator and a linear position sensitive detector after the sample. The resolution of scattering measurements performed in this way is necessarily low. In this specific case it is of $150^{\prime \prime}$ as compared to $10^{\prime \prime}$ in figure 5 . The advantage is that a large scattering-angle window is covered simultaneously and more intensity is available for the 
measurement. For comparison one can note that scattered intensity may be detected in the angular interval from $\sim 10^{\prime \prime}$ to $\sim 200$ " in the high resolution data of figure 5 , whereas scattered intensity may be detected in the angular interval from $\sim 150$ " to $\sim 5000^{\prime \prime}$ in the low resolution arrangement. An essential feature of the low resolution arrangement is its ability to do scattering measurements well above the critical angle $\theta_{c}$ [5]. This is of course not possible with the high resolution arrangement.

The high resolution scattering data in figure 5 shows that essentially no scattering takes place at or below the critical angle. This is consistent with the data in figure 6 , where the deviation between the Fresnel curve and the specular reflectivity data only shows up well above $\boldsymbol{\theta}_{\mathrm{C}}$. In the angular interval where the deviation is significant one observes consistently scattering away from the specular direction in the position sensitive detector. Fitting the data in figure 6 to equation (1) gave a r.m.s. surface roughness of $18 \AA$.

One important point made by Church [17] concerning the use of equation (1) to fit the specular reflectivity data is the fact that the surface wavelength bandwidth contributing to the scattering depends on the incident angle and a strong dependence of the r.m.s. surface roughness on the surface wavelength bandwidth have been reported [18-19]. Consequently the single number for $\sigma$ emerging from the use of equation (1) is generally to be taken as an effective roughness and not representing a meaningful physical quantity.

Data on the specular reflectivity of $\mathrm{SiC}$ at other energies $(80 \mathrm{eV}-1000 \mathrm{eV})$ have been obtained by Yanagihara et al. [13]. These data have also been fitted with the Fresnel curve modified with the first order scattering correction term (as in Eq. (1)). Their results for the r.m.s. surface roughness values were $18 \AA$ and $28 \AA$ for two different substrates. These values were in good agreement with independent laser interferometric measurements.

Alternatively the reduction of the specular reflectivity may be interpreted in terms of a diffuse interface $[9,10,11]$ where the interface could be the material itself [5] or a surface contamination layer. The problem with this model is that it does not predict any scattering. It seems that neither of the two explanations given above are very satisfactory and much work need to be done on the issue of surface contamination and the role of roughness on very short length scales [6] ( $\AA-\mu$-range).

\section{Multilayer defect study by high resolution X-ray diffraction.}

Grazing incidence X-ray diffraction experiments using $\mathrm{FeK} \alpha$ and $\mathrm{CuK} \alpha$ radiation have been performed in numerous cases [20] to study the perfection of the multilayer structure. The large majority of these experiments are low resolution studies, where a monochromatic and well collimated incident beam is generated using one or more crystal monochromators and/or slits but the detector system for the reflected/scattered beam is in most cases poorly collimated. This kind of measurements may be appropriately referred to as integrated reflectivity measurements. A review of X-ray diffraction studies of multilayered media is given by Nevot elsewhere in this series so I will not give a detailed review of these studies, rather I will describe the few high resolution

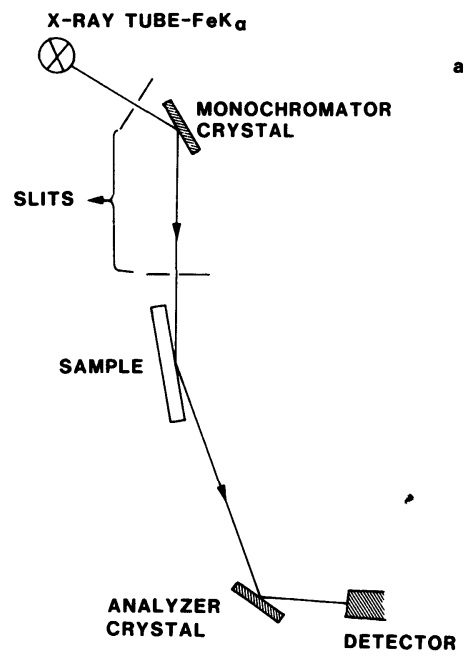

REAL SPACE

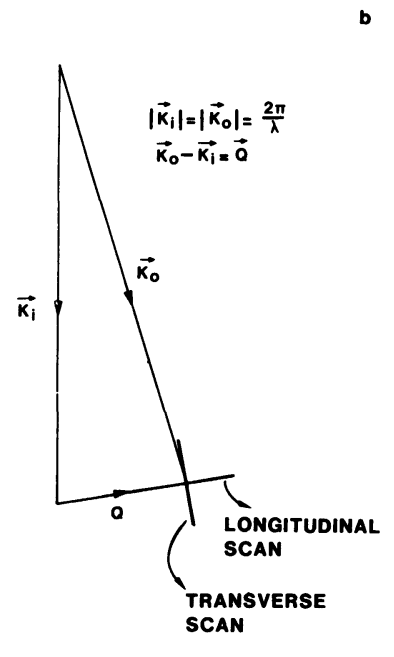

RECIPROCAL SPACE

Fig. 7. - High resolution X-ray diffraction arrangement for the study of the perfection of the structure of multilayers : (a) real space ; (b) reciprocal space. 
grazing incidence diffraction experiments which have been performed to date on multilayers.

The difference between integrated reflectivity and high resolution experiments is the addition of a narrowly collimated detection system. As in the case of the surface scattering experiment, this is achieved by using an analyser crystal to provide a balanced collimation a few arcsec wide on both sides of the sample. The arrangement is shown in figure 7 . Its performance is described in reference [1]. The arrangement is a classical triple-axis $\mathrm{X}$-ray diffraction set-up. The smallness of the Bragg angle of the multilayers allows one to obtain a very high resolution with the arrangement in figure 7 . Ishikawa et al. [8] obtains the same high resolution by using a premonochromator, in a dispersive setting, in their high resolution scattering arrangement. Resolution properties of a triple-axis X-ray diffractometer are discussed in references [15] and [16]. The measurements reviewed here were done at FeK $\alpha$. The diffractometer in figure 7 can be operated in two different modes. The first is a mode where the sample crystal alone is rotated through its reflection range - a socalled transverse scan [21]. It is called so because it is a scan perpendicular to the average reciprocal lattice vector of the sample, when depicted in reciprocal space (see Fig. 7b). The second mode is a mode where the sample and the analyser crystal is rotated in a $1: 2$ relation - a longitudinal scan [21] (see Fig. 7b), where the scan is parallel to the average reciprocal lattice vector of the sample. In the transverse scan one measures the angular distribution of the layernormal over the illuminated spot (mosaicity). In the longitudinal scan one measures the reflectivity curve from a parallel set of layers in the sample. Likewise $d$-spacing variations show up in the longitudinal scan.

\section{Data from W/Si-multilayers.}

Two examples of transverse and longitudinal data are shown in figures 8 and 9 along with the total integrated reflectivity measurement obtained by detecting all the reflected intensity in a wide open detector after the sample. The two multilayers were W/Si multilayers deposited on Si-wafers. Thickness

Table I.

\begin{tabular}{|c|c|c|c|c|}
\hline Composition & Code & Source & $\begin{array}{l}\text { Layer } \\
\text { period } \\
d \text { in } \AA\end{array}$ & $1 d_{\mathrm{w}}$ in $\AA$ \\
\hline $\mathrm{W} / \mathrm{Si}$ & OCES-100A & $\operatorname{ECD}\left({ }^{1}\right)$ & 52 & 9.76 \\
\hline $\mathrm{W} / \mathrm{Si}$ & OCES-045A & ECD & 23.3 & 3.32 \\
\hline
\end{tabular}

(1) Ovonic. Energy conversion devices. of individual layers and other parameters are listed in table I. Data are taken from reference [15].

Note that the wiggle structure which is evident in the longitudinal scans does not appear in the total integrated reflectivity measurements. This wiggle structure is related to the finite number of layers in the multilayers. The reason for the disappearance of the wiggles in the total integrated reflectivity scans is clearly seen in the transverse scans, where the width of these curves is a signature of a $20-30$ mdeg. wide distribution of the layernormal over the illuminated spot which is $10 \times 2 \mathrm{~mm}^{2}$. Since this width is nonnegligible in comparison with the width of the longitudinal scan one measures in the total integrated reflectivity scan a mixture of the reflectivity curve of parallel layers and the layernormal distribution, which results in a smearing of the wiggle structure [22]. In few cases total integrated reflectivity data have been obtained where the width of the reflectivity curve from parallel layers is large compared to the width of the layernormal distribution [23]. In these cases the wiggle structure is apparent also in the total integrated reflectivity scan. The full line in the longitudinal scan of figure 9 is a fit to the data assuming a perfect structure with no interdiffusion/roughness of layers. Basically only the density of the two materials and the relative thickness of the two materials have been varied [22]. Introducing any of the favoured defects [24], such as interfacial roughness/interdiffusion and $d$-spacing variations, provides, in this unique case, worse fits. The large amplitude wiggles could, however, still not be explained by the perfect layering model. This feature is observed in several cases [22] and remains an open question.

Ishikawa et al. [8] performed, in their study of scattering from the top of a W/C-multilayer, the analogue of a transverse (mosaicity) scan at the first order Bragg-peak. The transverse scan resembles to a large degree the scattering from the bare substrate. This is direct evidence that the layers follow the large scale figure error of the substrate. The analogue of a longitudinal scan was not performed.

Let me add that one other high resolution grazing incidence diffraction study of multilayers has been reported previously [25], but the multilayers studied in this case did not show the same state of perfection as reported in [22].

\section{Conclusion.}

High resolution X-ray diffractometry provides a powerful tool for the study of X-ray optical elements such as surfaces, substrates and layered periodic structures. In the present context it reveals that one of the major defects of multilayers is the large scale figure error of present day substrates such as float glass or Si-wafers. Future studies of multilayers on 

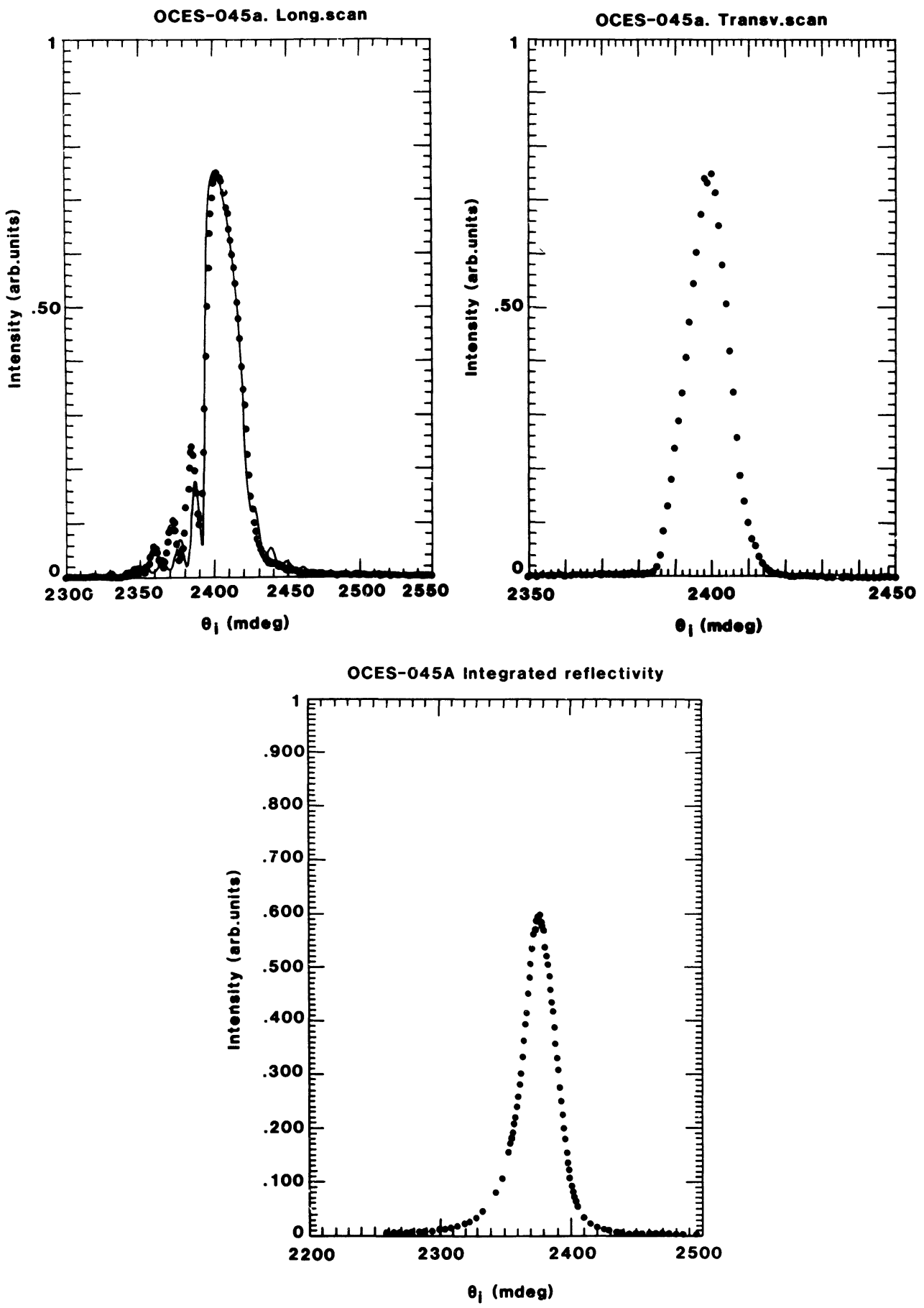

Fig. 8. - Integrated reflectivity and longitudinal and transverse scans for a W/Si multilayer (OCES-045A). See table I for details.

supersmooth substrates such as $\mathrm{SiC}$, will be very interesting and disclose whether or not the inadequacy of the substrate also influences the perfection of the layer structure as a whole.

\section{Acknowledgements.}

The author would like to thank the following for inspiring cooperation: A. Hornstrup, H.W. Schnopper, P. Dhez, E. L. Church and J. M. André. Further I would like to thank Ovonics-E.C.D. and Ferranti-Astron for their cooperation. Also I would like to thank P. $\varnothing$. Christensen and E. Jakobsen for technical assistance. Finally I thank an anonymous referee for clarifying comments. 

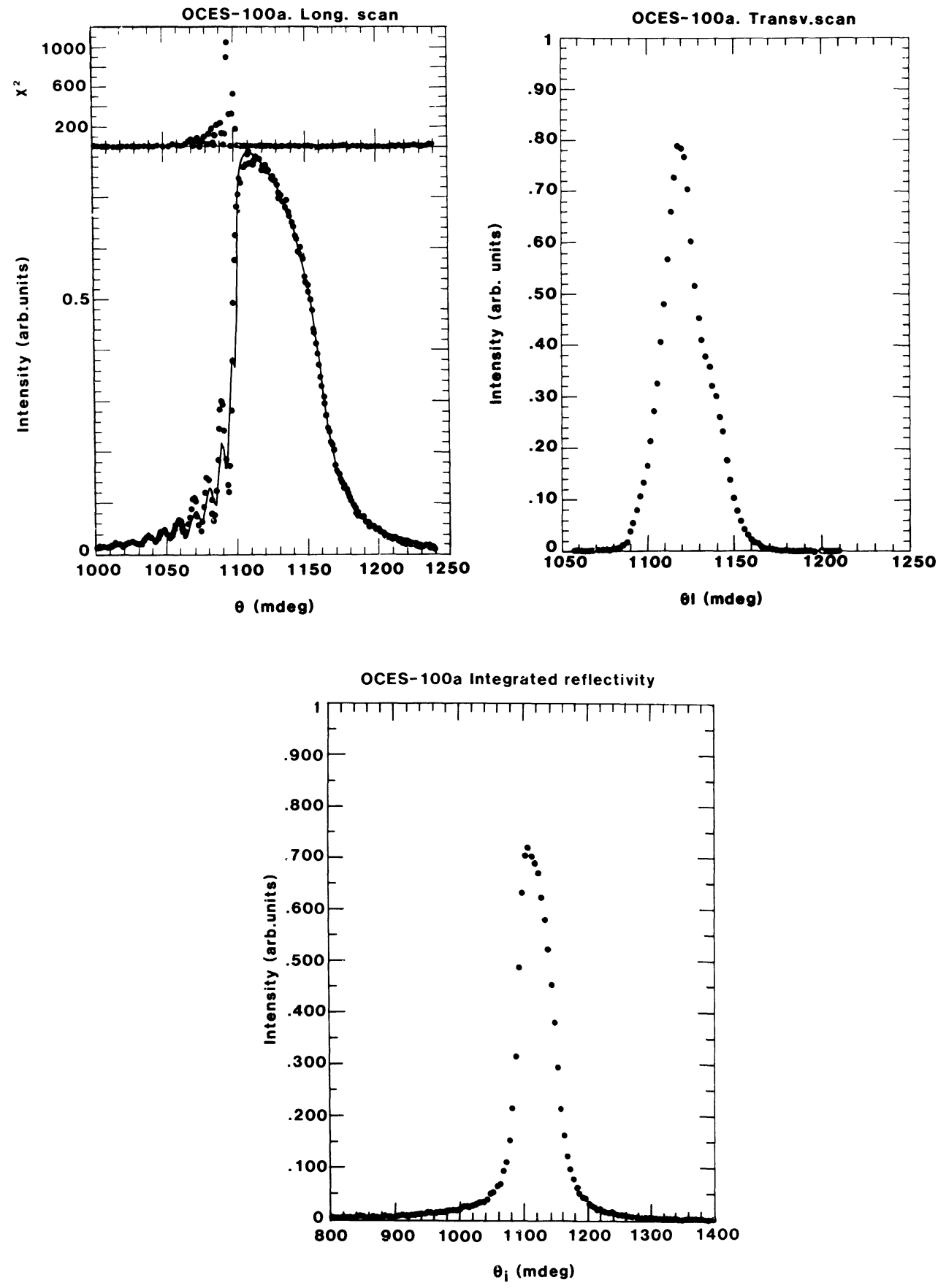

Fig. 9. - Integrated reflectivity and longitudinal and transverse scans for a W/Si multilayer (OCES-100a). See table I for details.

\section{References}

[1] Christensen, F. E., Hornstrup, A., Jacobsen, E., Jonasson, P., MADSEN, M. M., SCHNOPPer, H. W., Westergard, N. J., Ørup, P., A versatile three/four Crystal X-ray diffractometer for X-ray optical elements. Performance and applications, Nuclear Instrum. Methods A 256 (1987) 381-392.

[2] Matsushita, T., Ishikawa, T., Kohra, K., High resolution measurement of Angle-Resolved X- ray Scattering from Optically Flat mirrors, $J$. Appl. Crystallogr. 17 (1984) 257-264.

[3] Mancini, D. C., Bilderback, D. H., X-ray Mirror surfaces Evaluated by an X-ray Topographical technique. Nucl. Instrum. Methods A 208 (1983) 263-272.

[4] Hornstrup, A. and Christensen, F. E., Previously unpublished data. 
[5] Christensen, F. E., Hornstrup, A., Schnopper, H. W., Surface correlation function analysis of high resolution scattering data from mirrored surfaces obtained using a triple axis X-ray diffractometer. To appear in proc. S.P.I.E. (conf. in San Diego, August 1987) on Grazing incidence optics for Astronomical and Laboratory applications, and in Appl. Opt.

[6] Alehyane, N., Arboui, M., Barchewitz, R., ANDRÉ, J. M., Christensen, F. E., Hornstrup, A., Palmari, J., Rasigni, M., RIVOIRA, R., RASIGNI, G., X-UV and X-ray scattering measurements from a rough crystal surface characterized by electron micrography, submitted to J. Appl. Optics.

[7] KLos, R., X-ray scattering theories, S.P.I.E. 597 (1985) 135-144.

[8] Ishikawa, T., Iida, A. and Matsushita, T., Measurement of angle-resolved X-ray scattering from synthetic multilayers, Nucl. Instrum. Methods A 246 (1986) 348-351.

[9] Parrat, L. G., Phys. Rev. 95 (1954) 359.

[10] Nevot, L., Croce, P., Caractérisation des Surfaces par Réflexion Rasante de Rayons X. Application à l'Etude du polissage de quelques Verses Silicates, Rev. Phys. Appl. 15 (1980) 761 ;

BILDERBACK, D. H., Reflectance of X-ray mirrors from 3.8 to $50 \mathrm{keV}$, S.P.I.E.-proc. 315 (1981) 91.

[11] Als-Nielsen, J., Solid and liquid Surfaces studied by Synchrotron X-ray diffraction, Appearing in Structure and Dynamics of surfaces 2 (Springer Verlag) 1986.

[12] Compton, A. H., Allison, S. K., X-rays in theory and experiment (1935).

[13] Yanagihara, M., Niwano, M., Koide, T., Sato, S., Miyahara, T., Iguchi, Y., Yamaguchi, S., SASAKI, T., Soft X-ray reflection from SiC, TiC and WC mirrors, Appl. Opt. 25 (1986) numéro 24.
[14] Church, E. L., TAKaCs, P. Z., The interpretation of glancing incidence scattering measurements, S.P.I.E.-Proc. (1980) 640 and references therein.

[15] Christensen, F. E., Studies of Nematic to SmecticA Phase Transitions using Synchrotron Radiation. Experimental techniques and Experiments, Ph. D.-Thesis (1981).

[16] Pynn, R., FujiI, Y., Shirane, G., The Resolution function of a Perfect-Crystal Three-axis Spectrometer, Acta-Crystallogr. Sect. A 39 (1983) 38.

[17] Church, E. L., Private communication.

[18] Christensen, F. E., Singh, K. P., Schnopper, $\mathrm{H}$. W., Measurements of surface scattering from mirrored surfaces using a triple axis X-ray spectrometer, S.P.I.E. 597 (1985) 119.

[19] Hasinger, G., Die Streuung von Röntgenstrahlen an Polierte Oberflächen, Ph. D.-theses M.P.I. (May 1980).

[20] Applications of Thin-Film-Multilayered Structures to Figures X-ray Optics, S.P.I.E.-proc. 563 (1985).

[21] Christensen, F. E., Singh, K. P., Westergaard, N. J., SCHNOPPER, H. W., Measurements of mosaicity and perfection of crystallites in commercially available crystals using a 4-axis perfect crystal X-ray diffractometer, Appl. Opt. 26 (janvier 1987) numéro 2.

[22] Christensen, F. E., Hornstrup, A., Schnopper, H. W., High resolution X-ray diffraction studies of Multilayers, to appear in J. App. Crystallogr.

[23] Bruijn, M. P., Chakraborty, P., Van Essen, H., Verhowen, J., VAN Der Viel, M. J., Bartels, W. J., E-beam evaporated multilayer Soft X-ray coatings, analyzed with Cuk $\alpha$-radiation, S.P.I.E.-proc. 503 (1985) 182.

[24] Bruis, M. P., Multilayer X-ray coatings, Ph. D.Theses (1986).

[25] Warbuton, W. K., ReK, Z. U., Barbee, T. W. Jr, Performance tests on layered synthetic microstructures for X-ray optical elements, sympos. $\mathrm{X}$-ray Microscopy (Goettingen, West germany) septembre 1983. 